\title{
Tratamento da síndrome compartimental: Artigo de atualização
}

\author{
Treatment of compartiment syndrome: Update article \\ Tratamiento del síndrome compartimental: Artículo de actualización
}

Paula Lavigne de Sousa Costa ${ }^{1 *}$, Andrey de Almeida Carneiro², Amanda Freitas Teixeira da Silva ${ }^{1}$, Bernardo Felipe Santana de Macedo', Matheus Benedito Sabbá Hanna ${ }^{1}$, Caroline Lobato Pantoja', Fábio Brito Braga ${ }^{3}$.

\section{RESUMO}

Objetivo: Apresentar, descrever e discutir os achados da literatura referentes ao tratamento da síndrome compartimental. Método: Os dados foram coletados mediante revisão da literatura utilizando os descritores: síndrome compartimental, tratamento da síndrome compartimental; e como base em artigos publicados nos últimos 5 anos nas bases de dados Scientific Electronic Library (Scielo), Lilacs, MedLine e Pubmed. Como critérios de inclusão foram estabelecidos artigos originais e de revisão publicados na íntegra na forma on-line nos idiomas português, espanhol e inglês no período de 2014 a 2019, utilizando os descritores supracitados nos respectivos idiomas. Incluindo-se também artigos fora do período supracitado desde que considerados extremamente relevantes. Como critérios de exclusão utilizou-se artigos publicados fora do período estabelecido, artigos que não tratavam diretamente da síndrome compartimental e que foram considerados com informações repetitivas. Resultados: Foram coletados 80 artigos científicos, nos quais 40 trabalhos dentro dos critérios de inclusão. Conclusões: A fasciotomia persiste como o método mais efetivo para o tratamento da síndrome compartimental, porém mais pesquisas devem ser desenvolvidas na busca por prover novos meios de reduzir os efeitos adversos da SC.

Palavras-chave: Síndrome compartimental, Fasciotomia, tratamento não cirúrgico.

\begin{abstract}
Objective: Present, describe and discuss the findings of the literature regarding compartment syndrome treatment. Methods: The data were collected by literature review using the descriptors : "compartment syndrome", "treatment", as a basis for articles published in the last 5 years on Scientific Electronic Library (Scielo), Lilacs, MedLine and Pubmed. As inclusion criterion were established original articles and of review published in full, online, in portuguese, spanish and english, on period of 2014 to 2019, using the descriptors above mentioned. Results: Were collected 80 scientific articles, in which 40 works inside the inclusion criterion. Articles outside the aforementioned period have also been included since they are considered extremely relevant. Conclusion: Fasciotomy is still the most effective method for the treatment of comorbid syndrome, but further research should be developed to establish new effective way to reduce adverse effects of compartment syndrome.
\end{abstract}

Key words: Compartiment syndrome, fasciotomy, non-surgical treatment.

\footnotetext{
${ }^{1}$ Centro Universitário da Faculdade Metropolitana da Amazônia (UNIFAMAZ), Belém-Pará.

*E-mail: paulalavigne9@hotmail.com

${ }^{2}$ Centro Universitário do Pará (CESUPA), Belém-Pará.

3 Universidade do Estado do Pará (UEPA), Belém-Pará.
}

SUBMETIDO EM: 6/2019

ACEITO EM: 7/2019

PUBLICADO EM: $8 / 2019$ 


\section{RESUMEN}

Objetivo: Presentar, describir y discutir los hallazgos de la literatura referentes al tratamiento del síndrome compartimental. Método: Los datos fueron recolectados mediante revisión de la literatura utilizando los descriptores: síndrome compartimental, tratamiento del síndrome compartimental; y como base los artículos publicados en los últimos 5 años publicados en la base de datos Scientific Electronic Library (Scielo), Lilacs, MedLine y Pubmed. Los criterios de inclusión fueron establecidos artículos originales y de revisión publicados en su totalidad en el formulario en línea en portugués, español e Inglés en el período comprendido entre 2014 2019, utilizando los descriptores antes mencionados en sus respectivos idiomas. También se incluyeron artículos fuera del período antes mencionado desde que se consideraron extremadamente relevantes. Resultados: Se recolectaron 80 artículos científicos, en los cuales 40 trabajos dentro de los criterios de inclusión. Conclusiones: La fasciotomía persiste como el método más efectivo para el tratamiento del síndrome compartimental, pero más investigaciones deben ser desarrolladas en la búsqueda por proveer nuevos medios de reducir los efectos adversos de la SC.

Palabras clave: Síndrome compartimental, fasciotomía, tratamiento no quirúrgico.

\section{INTRODUÇÃO}

A Síndrome compartimental (SC) é definida como qualquer elevação na pressão intersticial dentro do compartimento ósseo-fascial, assim como da elevação da pressão intra-abdominal (PIA), causando elevada morbidade e mortalidade (VOLKMANN R, 1881). Suas consequências são diversas, cursando desde a perda da função de um membro, amputação e óbito. Sua prevalência é difícil de ser definida, pois muitas vezes não são diagnosticados durante a fase inicial da síndrome. Os atletas constituem a porção da população com maior incidência dessa patologia, sendo as mulheres dez vezes mais afetadas que os homens (DONALDSON $\mathrm{J}$, et al, 2014).

A elevação da pressão dentro de um compartimento osteomuscular ou abdominal fechado gera o comprometimento microvascular e pode evoluir com hipóxia e necrose tecidual. Assim, apesar de trata-se de um processo local, pode também gerar consequências sistêmica, como por exemplo, hipovolemia, rabdomiólise, desequilíbrio hidroeletrolítico e ácido-basico, falência renal e morte (LAWENDY AR, et al., 2016). Faz-se necessário entender que há 2 tipos principais de SC: SC muscular, que ocorre dentro do compartimento miofascial; e SC abdominal, observada no interior do compartimento da cavidade abdominal, é uma evolução grave do aumento na pressão nesse compartimento abdominal (TWSACS, 2017). A SC muscular pode ser classificada em aguda ou crônica, de acordo com seu tempo de evolução (VOU KEUDELL AG, et al., 2015; SILVA APS, et al., 2015; BAUMFELD D, et al., 2017).

A síndrome compartimental tem como características de sua fisiopatologia a promoção do estresse oxidativo, um distúrbio baseado no desequilíbrio entre agentes oxidantes e antioxidantes, em favor a geração de radicais livre ou em detrimento da velocidade de remoção destes (SIES H, et al., 2017). Tais oxidantes, também denominados espécies reativas de oxigênio (ERO's) são instáveis e promovem grande dano celular, a partir de reações de óxido-redução com moléculas orgânicas, tais como fosfolipídios, proteína e DNA. Essas espécies são caracterizadas pela sua toxidade e capacidade de gerar injúria, caracterizando a síndrome de isquemia e reperfusão (SIR) (CAMPOS MTG, et al., 2017). Estes efeitos podem ser minimizados por técnicas que promovem tolerância a SIR, como o condicionamento isquêmico que consiste em ciclos alternados de Isquemia e Reperfusão para proteger o órgão dos efeitos negativos da IR, podendo ser feito antes (précondicionamento), durante (per-condicionamento) ou após (pós-condicionamento) o período isquêmico principal (HAUNSELOY DJ, et al., 2016; LEJAY A., et al., 2016).

O tratamento padrão ouro da SC fundamenta-se na fasciotomia, que consiste em procedimento cirúrgico em que a fáscia muscular é cortada para aliviar a pressão e tratar a perda circulatória. Entretanto diversas complicações podem derivar deste manejo cirúrgico, como infecções e dor intensa. Porém diversas complicações podem derivar deste manejo cirúrgico. Por isso, diversas pesquisas têm sido realizadas para 
alcançar métodos igualmente eficazes, porém que proporcionem uma melhor recuperação ao paciente e menor risco de complicações. Nesse sentido, este artigo buscar reunir os aspectos mais relevantes e atuais do tratamento da SC disponíveis na literatura médica, a fim de difundir o conhecimento da mesma.

\section{MÉTODOS}

Tal revisão foi construída baseando-se no método PRISMA, o qual é constituído por um check list de 27 itens, os quais buscam direcionar e padronizar o processo de construção de revisões de literatura (GALVÃO TF, et al., 2015). Os dados foram coletados mediante revisão de literatura utilizando descritores com base no DECs e MeSH, como: "Síndrome compartimental", "Fasciotomia", "Tratamento não cirúrgico". Além disso, esses descritores também foram combinados da seguinte maneira: Síndrome compartimental $A N D$ fasciotomia, Síndrome compartimental AND Tratamento cirúrgico, Síndrome compartimental AND Tratamento.

Utilizou-se como base de dados a Scientific Electronic Library (Scielo), Lilacs, MedLine, Pubmed. Como critérios de inclusão foram estabelecidos artigos originais e de revisão publicados na íntegra na forma on-line nos idiomas português, espanhol e inglês no período de 2014 a 2019, utilizando os descritores supracitados nos respectivos idiomas. Também foram incluídos artigos fora do período supracitado, desde que considerados extremamente relevantes pelos autores. Como critérios de exclusão utilizou-se artigos publicados fora do período estabelecido, artigos que não tratavam diretamente da síndrome compartimental e que foram considerados com informações repetitivas. Dissertações, capítulos de livros e manuscritos também foram excluídos, bem como artigos que não apresentaram no título, resumo ou texto o assunto abordado nesta revisão. Tal seleção de critérios de inclusão e exclusão justifica-se pela busca da construção de uma revisão bibliográfica atual com base em artigos disponíveis nos principais idiomas e nas principais bases de dados da literatura médica. Os autores reconhecem a existência de riscos de viés por parte dos mesmos durante a construção deste trabalho, assim como risco de viés provenientes dos autores dos artigos utilizados.

A seleção dos artigos a partir dos descritores citado foi realizada em 3 etapas: Etapa 1: Leitura dos títulos e exclusão daqueles que não se adequaram aos critérios de inclusão ou exclusão, Etapa 2: Leitura somente dos resumos dos artigos permitidos a partir da realização da etapa 1 e eliminação daqueles que não se encontraram adequados nos critérios de inclusão, Etapa 3: Leitura na íntegra de todos os artigos restantes das etapas anteriores.

\section{RESULTADOS/DISCUSSÃO}

Foram selecionados 80 artigos por meio dos descritores nas bases de dados citadas, dos quais apenas 40 foram mantidos a partir da análise dos critérios de inclusão e exclusão.

Figura 1 - Divisão dos artigos de acordo com o período de publicação

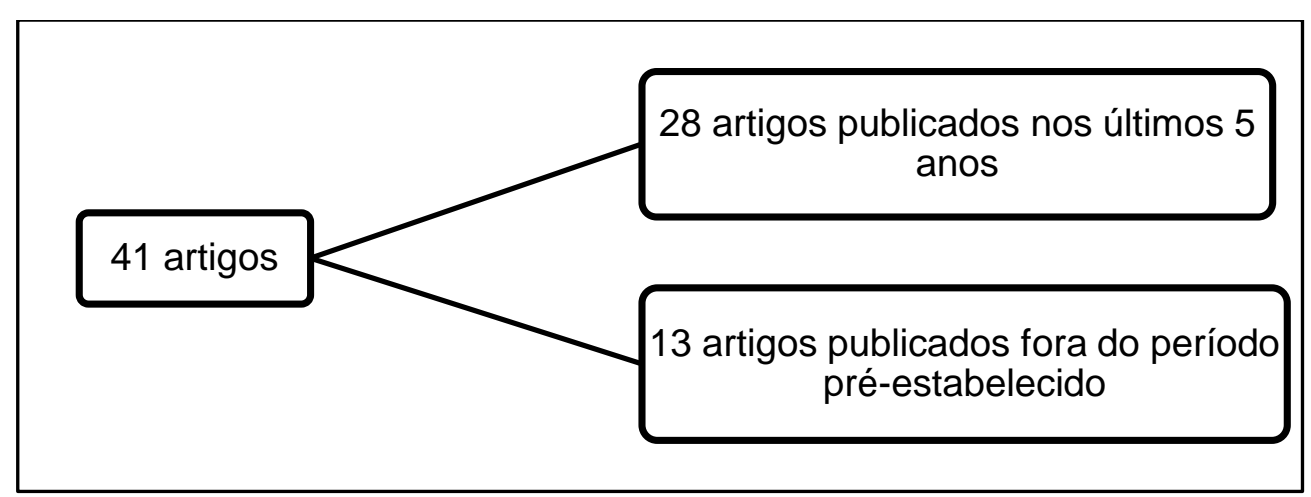

Fonte: Dados da pesquisa, 2019. 


\section{Tratamento Cirúrgico}

A pressão intracompartimental resulta em sofrimento tecidual com redução do fluxo sanguíneo e consequente necrose muscular. Desse modo, faz-se necessária a realização de procedimentos cirúrgicos descompressivos, os quais apresentam resultados mais eficazes em comparação com tratamentos não cirúrgicos (JAUREGUI J, et al., 2016).

A síndrome compartimental relacionado a sobrecarga relacionado ao esforço físico, a dor tende a desaparecer em repouso, porém o membro ainda poderá isquemiar na ausência de tratamentos (CRENSHAW $\mathrm{AH}, 1996)$. Por isso, a terapia é considerada fundamental e, em alguns casos, urgente.

Os primeiros cuidados a serem providenciados são a identificação e remoção de todas as forças compressivas externas, como hematoma, tumor e edema. É fundamental, também, a avalição precoce de hipovolemia, acidose metabólica, mioglobinemia a fim de identificar possível comprometimento renal. Administração de fluidos por via intravenosa e suplementação de oxigênio pode ser necessária durante o tratamento, assim como análise bioquímica do sangue e urinálise. Deve-se, também, assegurar a condição normotensa do paciente. $O$ adequado manejo de pacientes acometidos pela síndrome compartimental envolve uma atuação multidisciplinar podendo requerer a atuação de outras especialidades médicas como ortopedia (RAZA H, et al., 2015), cirurgia vascular (FRINK M, et al., 2009) e cirurgia plástica (DONALDSON J, et al., 2014).

Nesse sentido, destaca-se a fasciotomia (CONE J, et al., 2017; DAI AZ, et al., 2017; VOLETI PB, et al., 2015, POZZI A, et al., 2014), como método cirúrgico de escolha, que resulta na descompressão dos compartimentos musculares (CAMPOS MTG, 2018). Entretanto, há outros procedimentos que podem ser realizados, como endoscopia, mais recentemente, visando redução iatrogênicas, procedimentos minimamente invasivos. Além disso, diferente do que se acreditava alguns anos atrás, o valor da pressão compartimental no pré-operatório não tem valor prognóstico significativo (WINKES MB, et al., 2012; BIEDERT RM, et al., 1997; BOODY AR, et al., 2005).

O método de fasciotomia tradicional apresenta maior chance de infecção (BLAIR JÁ, et al., 2016) e longos períodos de processo cicatricial, de modo que o tempo de retorno as atividades cotidianas e que exigem maiores esforços é mais prolongada. Outros possíveis desfechos decorrentes do método tradicional de fasciotomia consistem em hematomas, lesão de nervos e vasos, adesão fascial e hemorragia do membro. Nesse sentido, Malffulli N, et al, 2015 publicou estudos que avaliaram a fasciotomia minimamente invasiva para tratamento da síndrome compartimental lateral ou anterolateral não traumática em atletas e seus resultados evidenciaram que $94 \%$ dos pacientes retornaram às atividades esportivas em 8 a 13 semanas.

Dentre técnicas minimamente invasivas presentes na literatura, Voleti PB, et al., 2015 descreveu a técnica de fasciotomia técnica endoscópica, que resultou em baixa taxa de complicações e recuperação rápida, permitindo o retorno de atletas ao esporte em pouco tempo. Enquanto Pozzi A, et al., 2014 descreveu uma fasciotomia assistida por endoscopia com uma mini-incisão.

Foi descrito na literatura que a liberação incompleta do compartimento fascial resultava em retorno dos sintomas em 44,7\% dos pacientes, então, Mathis KE, et al., 2015. Demonstrou que há uma forte relação entre o comprimento da fasciotomia e a diminuição da pressão dentro do compartimento. Estabeleceu, também, que deve haver liberação de pelo menos $90 \%$ do compartimento fascial acometido para retorno da pressão intracompartimental para níveis basais. Há relato, também, do emprego da sutura elástica como auxiliador no fechamento definitivo da incisão da fasciotomia que permitiu a sutura secundária e dispensou o uso de autoenxertia cutânea, técnica de manejo fácil e de baixo custo. Assim o procedimento se torna menos custoso e contribui para menor tempo de hospitalização quando comparada com a técnica a vácuo (CASTELO BRANCO PSM, et al., 2017).

A fasciectomia, em geral, tem melhores resultados que a fasciotomia. A fasciectomia pode ser realizada por meio de uma incisão longitudinal ou incisão transversal curta seguida de ressecção longitudinal de uma faixa da fáscia, utilizando para isso uma tesoura longa (ALMEIRA MJ, 2016). 
Enquanto Amputação é indicada em casos de diagnostico tardio, na qual não há função muscular e há trauma significativo no membro, ressaltando a importância da descoberta precoce da patologia (DONALDSON J, 2014). A SC no antebraço é pouco usual, mas pode ser vista em traumas automobilísticos, por exemplo. Os poucos estudos que pesquisaram os métodos de tratamento para tal concluíram que a fasciotomia e a fasciectomia foram igualmente efetivas (CROUZET P, 2009).

Quanto à Síndrome Compartimental Abdominal (SCA), considera-se a PIA normal entre 2 a $7 \mathrm{mmHg}$, sendo hipertensão intrabadominal acima de $12 \mathrm{mmHg}$. Enquanto valores acima de $30 \mathrm{mmHg}$ com falência de órgãos refratária às demais terapias já indica o tratamento cirúrgico de escolha, que consiste na laparatomia descompressiva (TWSACS, 2017; MALUSO P, et al., 2015).

A não descompressão resulta em má perfusão sanguínea intestinal, redução da circulação esplâncnica, insuficiência renal aguda pré-renal, diminuição do retorno venoso cardíaco e compressão da veia cava inferior, podendo resultar em acidose metabólica e colapso cardiovascular (MALUSO P, et al., 2015). O pós-operatório da SCA é caracterizado principalmente pela dor, logo uma analgesia adequada deve ser prescrita de modo regular. Além disso, o paciente deve ser monitorado cautelosamente devido possíveis complicações que podem vir a surgir, como rabdomiólise e falencia renal aguda (DONALDSON J, 2014).

\section{Tratamento Não Cirúrgico}

Diversos estudos têm sido realizados na busca por minimizar ou melhorar os traumas decorrente da cirurgia, como reduzir o tempo cirúrgico ou evitar infecções. As terapêuticas não medicamentosas mais eficazes são aquelas que evidenciam a parada do exercício ou diminuição da intensidade do mesmo, no caso de atletas. Entretanto, a dor tende a voltar gradualmente ao longo do tempo. Dentre os estudos realizados, testou-se o uso da fenilefrina e da dobutamina, as quais mostraram-se efetivas na elevação da pressão sanguínea quando usadas sequencialmente. Mostrou-se que a fenilefrina tem seu efeito diminuído ao longo do tempo, sendo necessário o uso da dobutamina após algumas horas, de modo que podem ser considerados como alternativa para o tratamento cirúrgico, pois possuem eficácia similar à fasciotomia (LIU D, 2015; LIU D, 2015). Outros artigos apontam o tratamento somente com uso de fisioterapia com Terapia Funcional Manual, o qual teve bons resultados.

Há o relato de emprego de medicamentos para o tratamento de SC classificada como leve na região do quadríceps, após contusão durante partida de futebol. Tratamento iniciado com bandagem compressiva para limitar expansão de edema, seguido de elevação da extremidade afetada e posteriormente a infusão intravenosa de metilprednisolona juntamente com analgésicos (paracetamol + matamizol). Evidenciou-se uma melhora clínica substancial com redução do edema e dor, permitindo flexão do membro em $70^{\circ}$. Assim em casos leves, a administração de corticosteróides intravenosos (prednisona $1 \mathrm{mg} / \mathrm{kg} / \mathrm{dia}$ ) pode ajudar a reduzir a inflamação,mas sempre sob observação médica e controle clínico frequente (PABLO-MÁRQUEZ B, et al., 2014).

Foi evidenciado também o uso da toxina botulínica A (BoNT-A) em pacientes com síndrome compartimental anterior ou anterolateral. As injeções da toxina foram guiadas por estimulação elétrica. Os autores concluíram que a BoNT-A reduziu a pressão intramuscular nos compartimentos envolvidos (RAJASEKARAN S, et al., 2016; HELMHOUT PH, et al., 2015; ISNER-HOROBETI ME, et al., 2013). Estudos tem sido feito buscando o manejo mais adequado para diminuir as consequências da SCA nos demais órgãos. Nesse sentido, comprovou-se que a melanocortina receptor agonista RO27-3225, já conhecida por suas propriedades anti-inflamatórias, é capaz de reduzir os danos cerebrais decorrentes da inflamação, reduzir o edema, produzir efeito neuroprotetor, além de reduzir o dano a outros órgãos como coração e pulmões (LIU D, et al, 2015).

\section{CONSIDERAÇÕES FINAIS}

A síndrome compartimental é uma doença complexa e de difícil diagnóstico em seu estágio inicial que pode se manifestar de forma aguda ou crônica. Esta síndrome é uma urgência médica que necessita de tratamento imediato, visando minimizar o risco de complicações, como em casos mais graves, que podem 
gerar a perda funcional de um tecido. Dessa forma, é consenso que a fasciotomia é o melhor método cirúrgico a ser desenvolvido nessas situações, mesmo diante de suas possíveis consequências. Entretanto, pesquisadores buscam por métodos menos invasivos e com menor chance de complicações. Porém, ainda há poucas evidências que sustentem a efetividade dos tratamentos não cirúrgicos quando comparados à fasciotomia. Dessa forma, é fundamental que pesquisas continuem a ser desenvolvidas visando o desenvolvimento de um método efetivo que evite as complicações decorrentes da cirurgia com objetivo de prover uma recuperação mais rápida e menos invasiva aos pacientes.

\section{REFERÊNCIAS}

1. ALMEIRA MJ. Síndrome do aprisionamento poplíteo e síndrome compartimental crônica dos membros inferiores: desafios no diagnóstico e tratamento. J Vasc Bras, 2016 15(4):265-267

2. BAUMFELD D, et al. Síndrome comparitmental aguda não traumática em atleta de futebol tratada por fasciotomia descompressiva segmentar: Relato de caso. Revista Brasileira de Ortopedia, São Paulo, 2017.

3. BEETSCH JW, et al. Xantine oxidase derived superoxide causes reoxigenation injury of ischemic cerebral endothelial cells. Brain. Res. v.786, p.89-95,1998.

4. BIEDERT RM, MARTI B. Intracompartmental pressure before and after fasciotomy in runners with chronic deep posterior compartment syndrome. Int J Sports Med. 1997;18(5):381-386.

5. BLAIR JA, et al. Infection and Nonunion After Fasciotomy for Compartment Syndrome Associated with Tibia Fractures. Journal of Orthopaedic Trauma, 2016, 30(7), 392-396.

6. BOODY AR, WONGWORAWAT MD. Accuracy in the measurement of compartment pressures: a comparison of three commonly used devices. J Bone Joint Surg Am. 2005;87(11):2415-2422.

7. CAMPOS MTG, LEME FOP. Estresse oxidativo: fisiopatogenia e diganóstico laboratorial. Pubvet, v.12, n.1, a10, p.18, Jan. 2018.

8. CARDEN DL, GRANGER DN. Pathophysiology of ischemia-reperfusion injury. J. Pathol. V. 190, p.255-66,2000.

9. CASTELO BRANCO PSM, et al. (2017). Sutura elástica no fechamento de fasciotomia para tratamento de síndrome compartimental associada à fratura da tíbia. Revista Brasileira de Ortopedia, 52(1), 103-106.

10. CONE J, INABA K. Lower extremity compartment syndrome. Trauma Surgery \& Acute Care Open, 2017.

11. CRENSHAW AH. Cirurgia Ortopédia de Campbell. 8ed. Manole; 1996; p.2033-2034, vol.3.

12. CROUTZET P, CHASSAT R, MASMEJEAN EH. Mini-invasive surgery for chronic exertional compartment syndrome of the forearm: a new technique. Tech Hand Up Extrem Surg 2009;13:137-40.

13. DAI AZ, et al. Open 4-Compartment Fasciotomy for Chronic Exertional Compartment Syndrome of the Leg. Arthroscopy techniques, 2017; 6(6), e2191-e2201.

14. PABLO-MÁRQUEZ B, et al. Síndrome compartimental aguda. SEMERGEN - Medicina de Familia, 2014. 40(4), 226228.

15. DONALDSON J, HADDAD B, KHAN WS. (2014). The Pathophysiology, Diagnosis and Current Management of Acute Compartment Syndrome. The Open Orthopaedics Journal, 8(1), 185-193. doi:10.2174/1874325001408010185

16. FERRARI R. Phatophysiological vs biochemical ischaemia: a key to transition from reversible to irreversible damage. Eur. Heart. J. Supp. v.3. p.2-10,2001.

17. FRINK M, et al. Compartment Syndrome of the Lower Leg and Foot. Clinical Orthopaedics and Related Research, 2009, 468(4), 940-950.

18. GALASSO G, et al. (2013). No-Reflow Phenomenon. Angiology, 65(3), 180 189.doi:10.1177/0003319712474336

19. HAUNSELOY DJ, YELLON DM. Ischaemic conditioning and reperfusion injury. Nature Reviews Cardiology, 2016, 13(4), 193-209.doi:10.1038/nrcardio.2016.5

20. HELMHOUT PH, et al. The Effectiveness of a 6-Week Intervention Program Aimed at Modifying Running Style in Patients with Chronic Exertional Compartment Syndrome: Results from a Series of Case Studies. Orthop J Sports Med, 2015.

21. ISNER-HOROBETI ME, DUFOUR SP, BLAES C, et al. Intramuscular pressure before and after botulinum toxin in chronic exertional compartment syndrome of the leg: a preliminary study. Am J Sports Med $2013 ; 41: 2558-66$.

22. JAUREGUI JJ, et al. (2017). Fasciotomy closure techniques. Journal of Orthopaedic Surgery, 25(1), 230949901668472.

23. LAWENDY AR, et al. Compartment syndrome causes systemic inflammation in a rat. Bone Joint J 2016; 98-B:1132

24. LEJAY A, et al. Ischemia reperfusion injury, ischemic conditioning and diabetes mellitus. Journal of Molecular and Cellular Cardiology, 2016, 91, 11-22. 
25. LIU C, et al. (2017). Reperfusion facilitates reversible disruption of the human blood-brain barrier following acute ischaemic stroke. European Radiology, 28(2), 642-649.doi:10.1007/s00330-017-5025-3

26. LIU D, et al. Melanocortin MC4 receptor agnoists alleviate brain damage in abdominal compartment syndrome in the rat. Neuropeptides 49, 2015, 55-61.

27. LIU D, et al. Melanocortin-4 receptor agonists alleviate intestinal disfunction in secondary intra-abdominal hypertension model. J Surgical Researches, 2015.

28. MAFFULLI N, et al. Single minimal incision fasciotomy for chronicc exertional compartment syndrome of lower leg. J Orthop Surge Res. 2016; 11(1):61.

29. MALUSO P, OLSON J, SARANI B. Abdominal Compartment Hypertension and Abdominal Compartment Syndrome. 2016; Critical Care Clinics, 32(2), 213-222. doi:10.1016/j.ccc.2015.12.001

30. MATHIS KE, et al. Effect of lower extremity fasciotomy length on intracompartimental pressure in an animal model of compartment syndrome: the importance of achieving a minimum of 90\% fascial release. Am J Sports Med. 2015; 43(1):75-8

31. POZZI A, et al. Single portal endoscopic treatment for chronic exertional compartment syndrome of the forearm. Tech Hand Up Extrem Surg 2014; 18:153-6.

32. RAJASEKARAN S, HALL MM. Nonoperative management of Chronic Exertional Compartment Syndrome: A systematic Review. Current Sports Medicine Reports, 2016.

33. RAZA H, MAHAPATRA A. Acute Compartment Syndrome in Orthopedics: Causes, Diagnosis, and Management. Advances in Orthopedics, 2015, 1-8.

34. SIES H, BERNDT C, JONES DP. Oxidative Stress. Annual Review of Biochemistry, 2017, 86(1), 715-748.

35. SILVA APS, et al. Síndrome Compartimental Crônica. Research Gate, São Paulo, Janeiro 2015.

36. THE WORLD SOCIETY OF THE ABDOMINAL COMPARTMENT SYNDROME (TWSACS). Abdominal Compartiment Syndrome Guidelines, 2017.

37. VOLETI PB, et al. Endoscopic Thermal Fasciotomy for Chronic Exertional Compartment Syndrome. Arthrosc Tech 2015;4:e525-9.

38. VOLKMANN R. Die ischämischen Muskellähmungen und Kontracturen. Centralblatt für Chirurgie. Leipzig 1881: 8: 801-83.

39. VON KEUDELL AG, et al. Diagnosis and treatment of acute extremity compartment syndrome. Lancet, 2015.

40. WINKES MB, ET AL. Compartment pressure curves predict surgical outcome in chronic deep posterior compartment syndrome. Am J Sports Med $2012 ; 40: 1899-905$.

41. YAMAKI IN, et al. "Kidney ischemia and reperfunsion syndrome: effect of lidocaine and local postconditioning." Revista do Colégio Brasileiro de Cirurgiões43.5 (2016): 348-353. 